\title{
Réécrire pour énoncer à nouveau : l'exemple de Ramuz chez Grasset
}

\section{Valentine Nicollier-Saraillon}

\section{(2) OpenEdition}

1 Journals

Édition électronique

URL : https://journals.openedition.org/genesis/1760

DOI : 10.4000/genesis. 1760

ISSN : 2268-1590

Éditeur :

Presses universitaires de Paris Sorbonne (PUPS), Société internationale de génétique artistique littéraire et scientifique (SIGALES)

Édition imprimée

Date de publication : 9 mai 2017

Pagination : 121-135

ISBN : 979-1023-105636

ISSN : 1167-5101

\section{Référence électronique}

Valentine Nicollier-Saraillon, « Réécrire pour énoncer à nouveau : l'exemple de Ramuz chez Grasset », Genesis [En ligne], 44 | 2017, mis en ligne le 17 mai 2018, consulté le 18 mars 2023. URL : http:// journals.openedition.org/genesis/1760 ; DOI : https://doi.org/10.4000/genesis.1760 


\title{
Réécrire pour énoncer à nouveau : l'exemple de Ramuz chez Grasset
}

\author{
Valentine Nicollier Saraillon
}

\begin{abstract}
La première objection qu'on se fait, c'est «À quoi bon?» Pourquoi refomenter un passé qui est définitivement sans doute refroidi, tisonner une cendre dont ne sait même pas si elle contient un reste de chaleur, chercher à faire reprendre vie à un petit feu qui a peut-être bien été un feu, mais ne l'est plus [?]*
\end{abstract}

$\mathrm{L}$ 'objection que se fait l'écrivain vaudois restera lettre morte. Ses lecteurs attentifs savent depuis longtemps qu'il est un écrivain doublé d'un réécrivain. Ramuz lit en effet systématiquement, plume en main, chacun de ses textes déjà parus avant toute nouvelle publication. La somme totale des différentes versions de ses vingt-deux romans est impressionnante : soixante-dix-huit (soit près de quatre par roman) selon la récente édition Slatkine (t. I-XIX, Genève, 2005-2013). Ce constat amène une conséquence terminologique : du point de vue de la génétique éditoriale comme de celui d'une histoire littéraire attentive à la matérialité de son corpus, aucun titre de Ramuz mentionné sans autre précision ne désigne autre chose qu'une famille de textes, ce qu'on pourrait appeler une exuvre; l'ignorer revient à risquer le malentendu 1 . L'interprétation de ce moment de la genèse - celui de la réécriture après édition - suppose bien sûr la comparaison des différentes versions d'une même œuvre et l'analyse de la quantité, de la nature et des effets de ces modifications ${ }^{2}$. Mais décrire ce geste particulier nécessite également de rendre compte des conditions dans lesquelles il s'exerce et de la représentation que s'en fait l'écrivain ${ }^{3}$. Je pense en effet que l'image que le scripteur donne et se donne de ce qu'il veut faire, fait ou pense avoir fait en réécrivant ses textes est une facette déterminante du processus de réécriture. C'est cette facette qu'il faut considérer avant tout si l'on veut comprendre pourquoi le «à quoi bon?» que Ramuz se lance ne le retient pas de réécrire.

\section{Les réécritures pour Grasset}

Cette recherche prend pour objet les quatorze romans que Ramuz publie chez Grasset. L'écrivain est sous contrat avec l'éditeur parisien depuis 1924. Excepté la «Lettre à Bernard Grasset» (1929), La Grande Peur dans la montagne (1926) et Une province qui n'en est pas une (1938), il ne lui donnera aucun inédit. Grasset reprend des titres déjà publiés, certains anciens, d'autres très récents, comme c'est le cas des romans que Ramuz fait paraître chez H.-L. Mermod, un industriel romand reconverti avec qui il est également sous contrat depuis début avril 19264. Pas de «campagne d'écriture clairement délimitée » ni de projet unique, comme c'est le cas de la longue révision à laquelle

(*) C.F. Ramuz, Journal 3, Euvres complètes, t. III, éd. D. Maggetti et L. Saggioratto, Genève, Slatkine, 2005, p. 327, entrée de novembre 1939.

1. Voir l'anecdote racontée par R. Mahrer au sujet des différentes fins de La Grande Peur dans la montagne, p. 7.

2. Parmi les critiques qui ont les premiers prêté attention aux réécritures éditoriales ramuziennes, citons A. Béguin, Patience de Ramuz, Neuchâtel, La Baconnière, 1950; G. Guisan, C.F. Ramuz ou le Génie de la patience, Genève, Droz, 1958; Th. Bringolf et J.-P. Verdan, Bibliographie de l'œuvre de C. F. Ramuz, Neuchâtel, La Baconnière, 1975. Pour des descriptions de cas précis de réécritures éditoriales, voir A. Pasquali, Genèse du récit. C. F. Ramuz. Adam et Ève, 2 vol., Paris, Minard, 1993; R. Mahrer, "Quand Ramuz remonte le courant - réécriture des romans en vue des Euvres complètes », Genesis, n 27, 2006, p. 107-127; V. Nicollier, «Une énonciation sous influence : Adam et Ève à Paris », Études francophones. Revue japonaise de didactique du français, 2007, p. 5-22. Récemment, les éditeurs des romans de l'écrivain dans la «Pléiade» (dir. D. Jakubec, 2005) comme ceux de ses Euvres complètes chez Slatkine (dir. R. Francillon et D. Maggetti) ont systématiquement pris en compte l'existence de réécritures éditoriales.

3. Voir ici même la contribution de R. Mahrer, rubrique «Enjeux».

4. Ce scénario fonctionne pour tous les romans parus après la signature du contrat avec Grasset, à l'exception de L'Amour du monde (1924) : La Beauté sur la terre, Farinet ou la fausse monnaie, Adam et Ève, Derborence mais aussi Terre du ciel (paru d'abord chez Georg/Crès). 
l'écrivain se livre lorsqu'il prépare ses Euvres complètes pour Mermod, mais un long compagnonnage qui dure depuis le premier contrat, jusqu'à la mort de l'écrivain en 1947. Ces réécritures, pourtant, gagnent à être envisagées dans leur ensemble. Bernard Grasset occupe en effet une place singulière parmi les éditeurs historiques de Ramuz. D'abord, l'écrivain ne s'était encore jamais lié de la sorte. Ensuite, partisan d'une diffusion efficace et de lancements en fanfare, Grasset mettra des moyens inédits pour promouvoir le Suisse romand. Il est véritablement celui qui, grâce au dévouement sans faille d'Henri Poulaille, chef de son service de presse dès 19245 , s'est chargé de faire connaître Ramuz aux Français. Enfin, les premières publications à l'enseigne de la «Maison» coïncident avec une intensification de la polémique sur le style de l'écrivain qui marque durablement ses rapports avec son éditeur. D'un côté un auteur accusé de mal écrire et qui, de plus, s'avérera un piètre investissement (en termes de ventes), de l'autre un éditeur grand public qui lui accorde tout de même sa confiance. Cette relation boitillante détermine le positionnement d'un Ramuz désolé de ne pas vendre et dès lors attaché à justifier son style vis-à-vis de ceux qui ont misé sur lui; elle engagera également l'écrivain à inclure la question du public dans son discours métapoétique, ce qui contribuera certainement à biaiser le regard porté par la critique sur le fruit de cette collaboration.

Les versions Grasset sont de fait peu considérées par la critique. Coincées quelque part entre les deux extrémités de la création, elles ne constituent un sommet remarquable ni pour les «romantiques», qui leur préfèrent l'édition originale, ni pour les «classiques», pour qui ces éditions ne sont qu'une étape sur le chemin de la perfection réalisée par l'édition ne varietur. Les versions intermédiaires souffrent pour la critique ramuzienne d'un autre défaut : elles porteraient les stigmates d'une certaine complaisance vis-à-vis du public parisien. Cette lecture date de la réception française de ces romans : au sujet de Joie dans le ciel, Robert de Traz se félicite que Ramuz offre au «grand public de langue française » du «simple» plutôt que du «précieux ${ }^{6}$. Les commentateurs récents, libérés de la problématique du bien écrire qui agitait les contemporains de Ramuz, conservent le motif mais pas l'enthousiasme : la taxe de douane qu' aurait consentie le Vaudois, parce qu'elle trahit le statut marginal de la littérature romande vis-à-vis du centre de consécration que représente Paris et qu'elle appauvrit la radicalité d'une poétique qui n'a de cesse de se justifier par son ancrage géographique et linguistique dans le terreau romand (que ce soit le lac, la campagne ou la montagne), est interprétée comme une compromission dans un art qui se doit de les mépriser, une indigne concession à une logique commerciale et à un besoin de reconnaissance par les pairs?

Pour les partisans de ce soupçon, l'accommodation au goût du public serait particulièrement évidente dans le travail sur le lexique, dont l'excentricité était la cible des critiques puristes. Les modifications de Ramuz auraient donc pour but d'ajuster sa langue avec la norme du français courant, en émoussant les étrangetés lexicales perçues comme diachroniques, diatopiques ou diastratiques. On sait pourtant que l'écrivain n'emploie pas de patois francoprovençal (désuet en Suisse romande depuis la seconde moitié du XIXe siècle) et se méfie du lexique régional, qu'il utilise avec parcimonie. Sa position, explicitée en 1929 dans la «Lettre à Bernard Grasset ${ }^{8}$ », est claire en 1914 déjà, période de son retour définitif en Suisse et du manifeste

5. Sur les relations entre les deux écrivains, lire M. Mermoud, «Henry Poulaille et C.F. Ramuz : salutations partisanes », dans C. Cerny (dir.), Henry Poulaille. Un écrivain au service de l'expression populaire, Bibliothèque cantonale et universitaire de Lausanne, 2005, p. 14-23. 6. Le Mercure de France, 15 décembre 1925, cité par G. Boillat, «L'accueil de Ramuz en France (1921-1926)», dans J.-L. Pierre (dir.), C.F. Ramuz 1, Paris, Minard, 1983, p. 75.

7. Les éditeurs contemporains, dans des termes proches de ceux de G. Guisan (C.F. Ramuz ou le Génie de la patience, op. cit.), évoquent un écrivain qui aurait «musel[é]» certains passages et qui aurait travaillé en vue de réaliser un "polissage stylistique», une «adapt[ation] à un public plus large », une «"normalisation" tendancielle du style», une syntaxe «assagie», etc. (C. Morzewski, «Un "mystère lémanique" », dans C. F. Ramuz, Romans 5, Euvres complètes, t. XXIII, Genève, Slatkine, 2012, p. 209-210 ; R. Francillon, «Introduction», dans C. F. Ramuz, Romans 2, Euvres complètes, t. XX, p. 7 ; S. Pétermann, «La beauté comme absolu », dans C.F. Ramuz, Romans 8, Euvres complètes, t. XXVI, Genève, Slatkine, 2013, p. 181). Le jugement négatif porté par la critique sur la valeur des versions Grasset influence jusqu'à l'appréciation de la genèse éditoriale des œuvres. Ainsi, lorsque Ramuz revient à l'édition originale pour préparer ses Euvres complètes chez Mermod au dépend de la version intermédiaire parue à l'enseigne de la «Maison», il fait le choix de l'intégrité (G. Boillat, «L'accueil de Ramuz en France (1921-1926)», art. cité, p. 75).

8. C. F. Ramuz, «Lettre à Bernard Grasset», dans Essais 2, Euvres complètes, t. XVI, Genève, Slatkine, 2009 [1929], p. 123-147. 
Raison d'être, dans lequel il signifie son refus du folklore et du pittoresque comme autant d'affiliations aux régionalistes et aux tenants d'un roman social' ${ }^{9}$. Ramuz souhaite éviter les effets de couleur locale et de didactisme; il mesure de fait ses efforts lexicaux relativement à un vraisemblable réaliste. Or, ses romans, même une fois publiés à Paris, ne cessent pour autant de se dérouler en Suisse romande : un fléchissement normatif du lexique serait, d'un point de vue poétique, contreproductif.

De fait, la prise en compte du lectorat de Grasset ne passe pas par le remplacement d'un lexique coloré par des mots bien français. Pour mesurer l'ampleur d'un tel lexique dans les romans de Ramuz, on peut partir du relevé effectué, selon des critères lexicographiques, par les éditions critiques récentes de la «Bibliothèque de la Pléiade» et de Slatkine : Aline nécessite vingt-huit notes explicatives, Aimé Pache, peintre vaudois quarante, La Guérison des maladies trente. Les mots appelant ces notes sont, dans un grand nombre de cas, maintenus dans les versions Grasset (pour citer deux exemples, vingt-trois expressions sur vingt-huit sont conservées dans la réécriture d'Aline; trente-six sur quarante dans celle d'Aimé Pache). Cette tendance s'observe dès le premier roman que Ramuz publie chez Grasset en 1924, La Guérison des maladies. Sur les trente expressions annotées par les éditeurs de «La Pléiade» et de Slatkine, en exceptant les occurrences supprimées avec un plus long segment dans un geste qui dépasse l'ajustement lexical ${ }^{10}$, seules trois font l'objet d'un remplacement terme à terme. «S'aheurter» devient «se heurter»; les locutions prépositionnelles traditionnelles «en bas du village», «en haut de la montagne» remplacent l'usage régional (mais pas strictement helvétique) sans de («en haut la montagne»); «la barque à pierres » devient « la barque de pierres ». Dans les deux premiers cas, Ramuz remplace en effet des expressions régionales par des expressions standardisées. Dans le dernier cas, il élimine le nom d'un type d'embarcation à deux mâts qui n'est plus guère utilisé en Suisse romande à partir du début du XXe siècle ${ }^{11}$. Trois sur trente : c'est peu. L'entrée lexicale et l'hypothèse de «correction» systématique effectuée par Ramuz ne rend donc compte que très partiellement du travail effectué par l'écrivain pour Grasset. L'intention de l'écrivain ne se résume pas à une adaptation de son vocabulaire en vue d'un nouveau lectorat. Du point de vue du résultat des réécritures, l'hypothèse puriste n'échoue pas seulement à donner une visibilité à ce qui bouge, elle empêche également de prendre la mesure de ce qui demeure. Or, à l'échelle d'un roman, quelques suppressions ne suffisent pas à rebattre les cartes lexicales et confèrent peut-être même une plus grande force à celles qui sont conservées.

\section{Les termes contractuels de la réécriture : «nouvelle composition», « réimpression » et «texte revu»}

Le contrat de 1924 engage Ramuz auprès de Grasset pour au moins cinq «compositions originales», dont une destinée aux «Cahiers verts» de Jean Halévy ${ }^{12}$. Un autre document daté du 30 janvier prévoit la «réimpression» de deux volumes, dont La Guérison des maladies (le second sera Joie dans le ciel). Ces documents ne se sont pas révélés très contraignants, car si Grasset commence bien par publier La Guérison des maladies, Ramuz ne lui donnera en définitive, comme on l'a vu, que peu de compositions originales, sans que cela ne nuise à leurs relations commerciales. L'écrivain bénéficie donc d'une marge de manœuvre qui, si elle lui permet d'exercer son droit d'auteur très librement, ne lui offre, on le verra, aucun avantage commercial. L'éditeur distingue en effet «composition originale» et «réimpression », mais il ne mentionne pas la troisième possibilité,

9. C.F. Ramuz, Raison d'être, dans Essais 1, Euvres complètes, t. XV, Genève, Slatkine, 2009 [1914], p. 35. Sur la position de Ramuz vis-à-vis du régionalisme, voir D. Maggetti, «Ramuz et le régionalisme», dans Au carrefour des cultures et des esthétiques, C. F. Ramuz 6, La Revue des lettres modernes, 1998, p. 77-92. À noter que la position de Ramuz sur le lexique s'inscrit de façon cohérente dans la représentation de l'écriture littéraire qui domine de 1890 à 1940, pour laquelle le travail de la langue ne passe plus par le choix des mots mais par leur ordre dans la phrase (G. Philippe, Sujet, verbe, complément. Le moment grammatical de la littérature française. 1890-1940, Paris, Gallimard, 2002).

10. «Le régent», «sauter contre», «mettre les choses au long», «branter», «coller» et «toute espèce de» font partie de larges segments entièrement supprimés.

11. La «barque à pierres » achemine sur l'autre rive du Léman des matériaux de construction et des pierres en provenance de la Savoie.

12. Pour plus de précisions sur le début des relations commerciales entre Ramuz et les gens de Grasset, lire G. Boillat, «L'accueil de Ramuz en France (1921-1926)», art. cité. 
intermédiaire du point de vue de l'originalité du texte, de la publication d'un «texte revu». C'est pourtant précisément ce que lui propose Ramuz.

La terminologie adoptée par Grasset embarrasse l'écrivain lorsqu'il doit désigner son travail. S'il qualifie publiquement les ouvrages publiés par l'éditeur parisien de «réimpressions », il fait à maintes reprises valoir dans sa correspondance son intention d'améliorer le texte original et le travail considérable que cela lui coûte. Par exemple, dans ce brouillon de lettre destiné à son éditeur :

Je vous envoie $[\ldots]$ deux volumes d'entre ceux qui pourraient être réimprimés : Guérisons des maladies et Terre du ciel (le premier paru fin 1917, le second fin 1921) [...] Je dois vous dire qu'il n'est jamais entré dans mes intentions de les faire paraître à nouveau sous leur forme primitive. J'aimerais pouvoir être en mesure d'apporter à l'un et à l'autre (grâce au «recul» dont je dispose) d'importantes modifications $[\ldots]^{13}$.

Même double discours (réécriture en coulisse, réimpression sur la scène) au sujet d'Aline : Ramuz défend en privé à Poulaille un texte «corrigé, re-corrigé, et re-re-corrigé14 » alors qu'il introduit publiquement l'ouvrage comme une «réimpression» :

\section{Mon cher Éditeur,}

Il faudrait qu'il fût bien entendu que c'est vous qui prenez la responsabilité de la présente réimpression. Moi, je me laisse faire; je ne sais plus, c'est trop vieux. Je ne vois pas en quoi cette «histoire» peut encore intéresser vos lecteurs. [...] J'ai donc remis mon sort entre vos mains, étant bien résolu, pour ce qui est de moi, à ne voir dans votre décision qu'un effet de votre extrême indulgence; - dont j' aurai du moins l'occasion de dire ici combien j'en ai été touché15.

Le terme de «réimpression» conforte le positionnement de Ramuz, qui sous-entend habilement qu'à 20 ans il était déjà le grand écrivain choisi par Grasset; il met également en évidence le défaut de visibilité de son travail de réécriture, qui n'est valorisé ni par l'éditeur, ni par le public qui ignore qu'il achète une «nouveauté 16 » et ne présente donc aucun intérêt commercial pour l'écrivain. Il est au contraire perçu par les gens de Grasset comme menaçant la bonne marche des affaires. Ramuz doit donc plaider en faveur des nouvelles versions. Pour parer les inquiétudes de son éditeur - relatives par exemple à des retards dus à certaines réécritures plus difficiles que prévues - et justifier l'intérêt d'une nouvelle version alors même que c'est l'ancienne qui a été choisie, il en vante le resserrement. Il décrit à Poulaille le «nettoyage» auquel il a procédé lors de la réécriture d'Aline; de même, avant de se lancer dans celle de La Beauté sur la terre, il confie à Pierre Tisné souhaiter «revoir et abréger sensiblement le texte», qu'il présente plus tard à Poulaille comme «considérablement resserré, nettoyé, condensé 17 ».

Dans la représentation du bon goût en littéracie que se fait Ramuz, less is more. L'argument n'est pas fallacieux. Contrairement à d'autres écrivains comme Proust ou Montaigne, connus pour relire en ajoutant du texte, Ramuz élague; c'est là une tendance générale que l'on remarque dès la première rédaction complète de ses projets

13. Brouillon de lettre de Ramuz à Grasset daté du 18 novembre 1923; Journal 3, op. cit., p. 147. Ramuz avait déjà essayé de régler le statut des rééditions à son avantage, comme on le voit lors des négociations précédant la signature du contrat avec Grasset. Au projet de contrat que lui fait parvenir L. Brun le 22 janvier 1924 et qui ne mentionne que la publication d'inédits, Ramuz répond en s'inquiétant de savoir si Grasset compte toujours publier La Guérison des maladies. Il profite de l'opportunité de la discussion pour proposer de compter ce «texte revu» comme un «inédit»: «Une des clauses du contrat stipule qu'il s'agirait de 5 volumes inédits. M. Grasset dans sa lettre du 20 décembre dernier avait bien voulu me dire être prêt à rééditer "Guérison des maladies" dont j'ai revu complètement le texte, qui est prêt. Ce volume serait-il compris dans le total ci-dessus? Le projet reste-t-il valable?» Grasset rassure Ramuz sur le sort des rééditions prévues mais il exclut de les considérer comme des inédits : «Bien entendu nous acceptons les 2 réimpressions qui viendront s'ajouter aux 5 inédits » (document ayant circulé entre Ramuz, Brun et Grasset entre le 22-30 janvier 1924, c'est Ramuz qui souligne; D. Maggetti et S. Pétermann, Vies de Ramuz, Genève, Slatkine, 2013, p. 102).

14. Lettre à Poulaille du 24 août 1926; Fonds privé. Je remercie Daniel Maggetti, directeur du Centre de recherches sur les lettres romandes, son collaborateur Stéphane Pétermann ainsi que Laura Saggiorato, de la Bibliothèque cantonale et universitaire de Lausanne, qui m'ont autorisé et facilité l'accès aux archives du fonds Ramuz.

15. C.F. Ramuz, «Avertissement», dans Romans 1, Euvres complètes, t. XIX, Genève, Slatkine, CD-Rom; je souligne.

16. À une exception près, celle de la réédition des Signes parmi nous en 1931, dont le texte se termine sur cette mention : «Écrit 1919. - Repris $1931 »$. Le livre corrigé en vue de cette réédition porte en effet la date de «juin $1931 »$.

17. Respectivement lettre à Poulaille du 5 mars 1928 (C. F. Ramuz, ses amis et son temps, t. VI, éd. G. Guisan, Lausanne/Paris, La Bibliothèque des arts, 1970, p. 171), à Tisné du 20 janvier 1928, puis à Poulaille du 4 août 1926; Fonds privé. 
et qui se poursuit au moment des relectures postérieures à la publication. Parmi les romans repris pour Grasset, peu échappent à la règle de la réduction 18 . Dans l'argumentaire que Ramuz destine à ses éditeurs, il assigne au resserrement une finalité méliorative, suivant un imaginaire de la belle langue qu'il professe auprès des gens de Grasset et qu'il prête aux lecteurs de la Maison :

[c]ette nouvelle version [toujours de La Beauté sur la terre] aura au moins 30 pages de moins que la précédente. J'ai fait tout ce qu'ai pu pour condenser, abréger, rendre clair, c'est-à-dire vous satisfaire et, j'espère, le public ${ }^{19}$.

Ramuz justifie son travail auprès de son éditeur en prêtant à sa recherche d'économie une vertu stylistique : la clarté, idéal langagier qui traverse les représentations du français au point de représenter le refuge privilégié de ses défenseurs 20 . Il suppose à son correspondant une conception de ladite clarté fondée sur le concis, le refus des développements inutiles et tourne ainsi le résultat d'un travail qui comporte aux yeux de l'éditeur de nombreux risques en un argument commercial dont il estime qu'il fera mouche auprès de son interlocuteur comme du public. Ce qui est surtout clair à nos yeux, c'est qu'il ne se réécrit pas pour satisfaire une obligation contractuelle, ni pour proposer un texte original. Son geste poursuit d'autres finalités.

\section{Ce que la réécriture fait à la communication littéraire}

On sait avec Vaillant que les conditions matérielles et symboliques de la communication littéraire influencent les genres pratiqués et qu'il arrive que les écrivains, répugnant à s'adapter, élaborent des formes transgressant le modèle de communication qui leur est imposé21. On fera l'hypothèse que cette transgression, que l'historien de la littérature traque dans les textes, se joue également dans les attitudes et les représentations qui les animent. Dans cette perspective, décrire la valeur que revêt pour un écrivain le geste de réécriture contribue à caractériser sa représentation d'un événement central dans le régime de la littérature-texte : la publication.

Depuis le premier tiers du XIX ${ }^{\mathrm{e}}$ siècle, le livre publié figure au centre d'une communication littéraire orchestrée par des éditeurs qui, d'artisans, sont devenus des acteurs de la vie culturelle. Il ne diffuse plus la parole d'un écrivain, mais un texte proposé par un éditeur. Ce texte-là doit son avènement à un événement, la publication, qui consacre la rupture du lien de productivité qui le lie à son créateur. Le scripteur, dont l'exercice de l'écriture prévoit déjà la disparition ${ }^{22}$, est promu au rang d'auteur, résultat d'une construction complexe qui, dès lors que l'édition est devenue un phénomène médiatique, déborde largement l'effet du texte ou de l'œuvre23. La publication conduit à une aggravation de cette distanciation entre le scripteur, le lecteur et l'écrit : jusque-là privé, unique et le plus souvent autographe, il devient public, imprimé, multiple. L'interprétation est désormais en partie le privilège des lecteurs ; quant à l'objet écrit, il n'est plus le produit d'un seul agent, mais celui d'un processus de fabrication impliquant plusieurs instances : l'auteur et l'éditeur, mais également le prote, le relecteur, le libraire 24 ... De nombreux écrivains racontent à quel point l'événement constitué par la publication de leur livre affecte la relation immédiate qu'ils entretiennent, comme producteurs, avec leur texte. Certains le déplorent, d'autres le considèrent avec indifférence voire

18. À l'exception d'Aline (dont l'ajout d'un «Avertissement» contribue à gonfler la taille de 1104 signes), d'Adam et Ève (+ 675 signes), de Derborence (+ 105 signes) et de Jean-Luc Persécuté (+ 77 signes), les versions Grasset sont plus courtes que celles à partir desquelles elles ont été travaillées. Le volume total de ces coupes varie entre quelques centaines et plusieurs dizaines de milliers de signes : Les Signes parmi nous (- 505 signes), Le Garçon savoyard (- 3874 signes), Terre du ciel (- 9581 signes), Aimé Pache (- 14382 signes), Farinet ou la fausse monnaie (- 21200 signes), La Beauté sur la terre (- 26363 signes) et La Guérison des maladies (- 39257 signes, soit un peu plus d'un cinquième de la première version...)

19. Lettre à Grasset du 1er mars 1928 ; Fonds privé

20. G. Philippe, Le Français, dernière des langues. Histoire d'un procès littéraire, Paris, PUF, 2010.

21. A. Vaillant, «Modernité, subjectivation littéraire et figure auctoriale», Romantisme, $\mathrm{n}^{\circ} 148-2,2010$, p. 11-25.

22. Une des caractéristiques généralement considérées comme définitoires du message écrit est la durabilité de son support, qui permet à sa production d'être effectuée en amont de sa réception, et donc à cette réception de se réaliser en l'absence du producteur du message.

23. J. Meizoz, Postures littéraires. Mises en scène modernes de l'auteur, Genève, Slatkine, 2007.

24. E. Souchier, «L'image du texte. Pour une théorie de l'énonciation éditoriale», Les Cahiers de médiologie, $\mathrm{n}^{\circ}$ 6, «Pourquoi des médiologues », 1998/2, p. 137-145. 


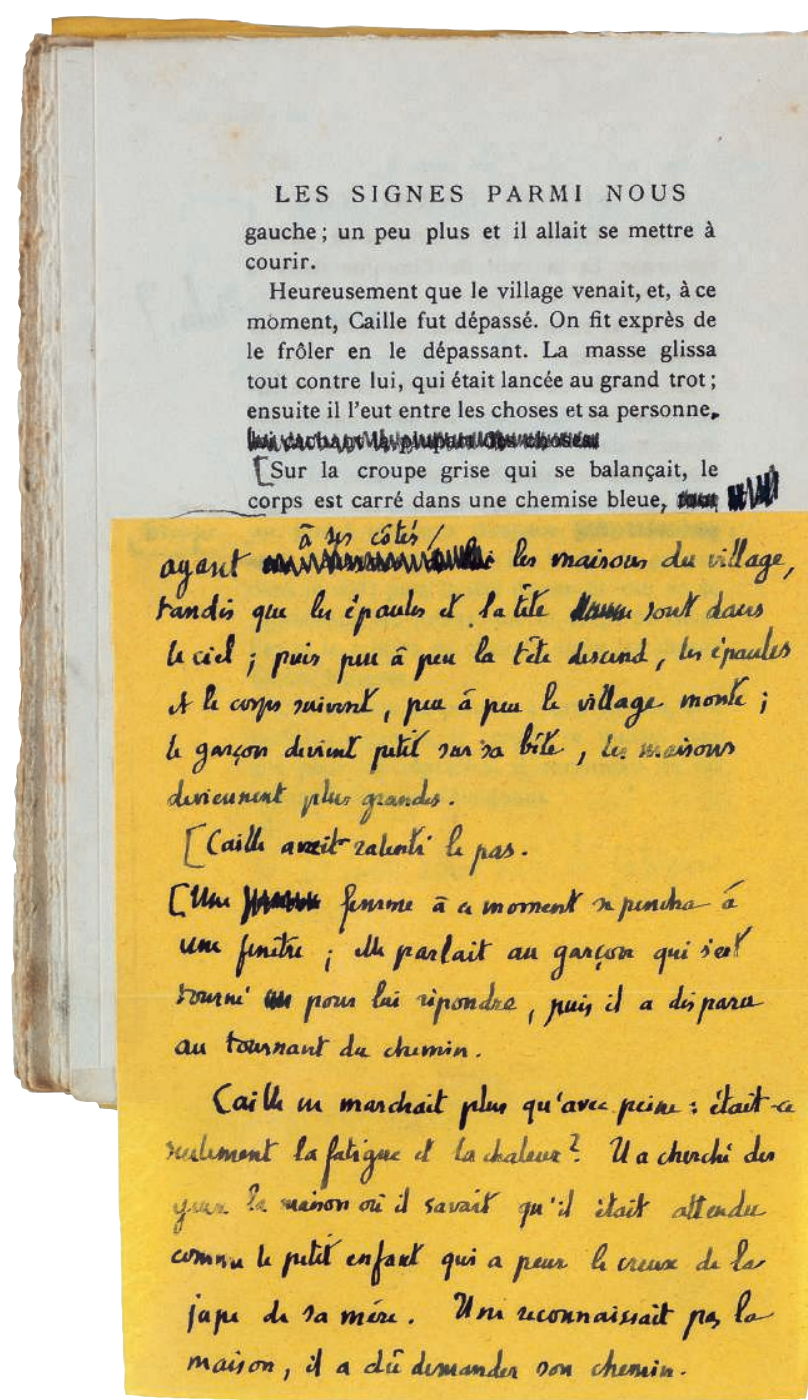

s'en réjouissent. Ramuz, on s'en doute, appartient à la première catégorie :

Eût-on fait une œuvre parfaite, on n'en saurait tirer aucune satisfaction. Toute œuvre, sitôt achevée, et par son achèvement même, devient votre pire ennemie. C'est un morceau de vous qui est mort quant à vous. C'est un morceau de vous qu'il faut que vous rejetiez résolument, puisque mort. Et il arrive qu'il puisse être vivant pour d'autres, mais c'est d'une vie à lui, indépendante de la vôtre et souvent contradictoire à la vôtre. D'où le danger ${ }^{25}$.

À ces écrivains-là se présentent plusieurs options : ne jamais entériner le passage du binôme «scripteur-manuscrit» au trinôme «auteur-livre-lecteur» (autrement dit, ne pas publier), envisager l'œuvre à venir comme la poursuite de l'écriture de l'œuvre précédente ${ }^{26}$, ou ne pas envisager la

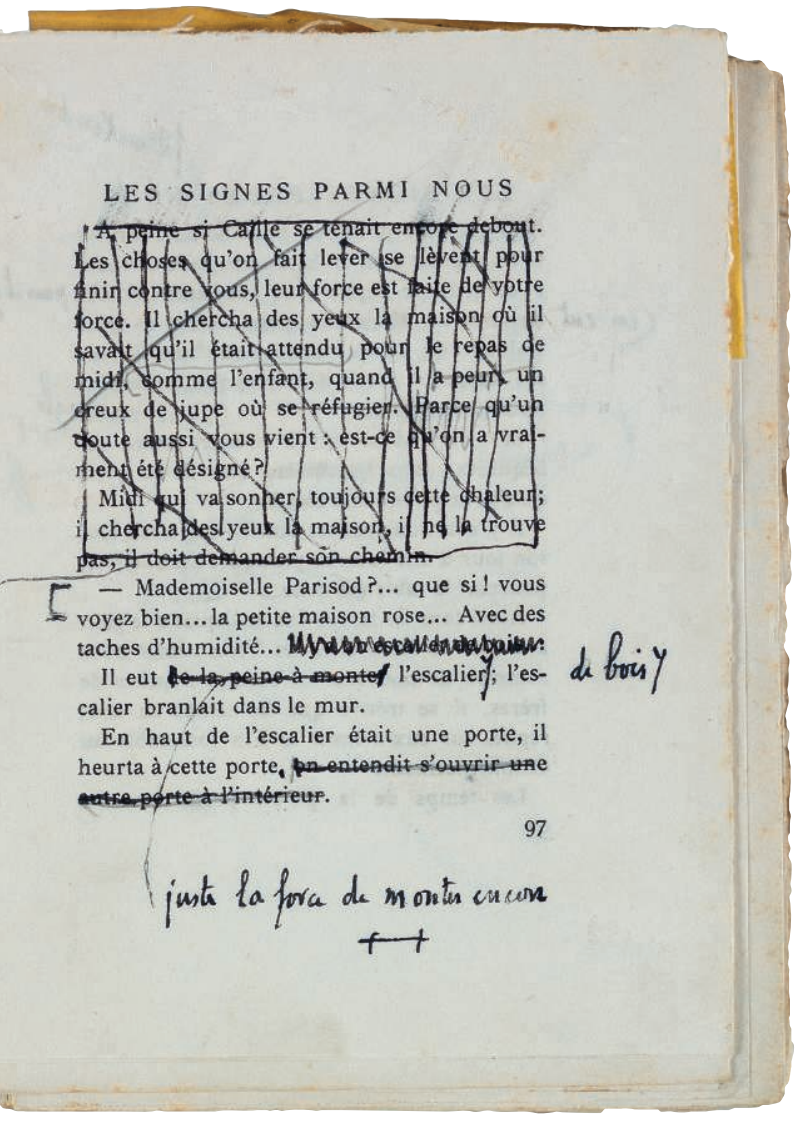

Fig. 1 : Pages de l'exemplaire de l'édition originale des Signes parmi nous (1919) corrigé par Ramuz en vue de la réédition du roman chez Grasset (1931) (D. Maggetti et S. Pétermann, Vies de C. F. Ramuz, op. cit., 2013, p. 50; Fonds privé)

publication comme la dernière étape de l'élaboration de l'œuvre. Les partisans de la dernière option contournent le rôle dévolu au livre dans le scénario éditorial moderne pour le traiter comme le maillon d'une chaîne de production qui s'allonge à chaque réédition (voir fig. 1).

La représentation que l'auteur se fait de la publication conditionne la possibilité de l'acte de réécrire. Or on

25. C. F. Ramuz, Journal 2, Euvres complètes, t. II, Genève, Slatkine, 2005, p. 2, 211, entrée du 9 décembre 1911.

26. Pour ceux-là, l'inachèvement de l'histoire se confond avec celle de son écriture. Par exemple, Frédéric Pajak, lors d'une lecture publique organisée à Lausanne le 17 mars 2015 suite à l'attribution du Prix suisse de littérature pour le tome IV du Manifeste incertain, répond à une question sur son projet d'ajouter cinq tomes à la série que son but n'est pas d'atteindre un certain nombre de volumes, mais d'écrire un livre qui ne se finit pas, «jusqu'à ce que mort s'ensuive». 
observe dans le cas de Ramuz un double déni : déni de la déprise auctoriale actée par la publication d'un livre, et, plus avant, déni du fonctionnement différé de la scène énonciative du message écrit. Le terme «déni»n'est pas choisi au hasard, car il ne s'agit pas pour l'écrivain d'échapper aux contraintes réelles de l'écriture littéraire mais bien, au contraire, de les utiliser à son avantage. On peut ainsi qualifier de braconnage la réécriture d'un texte littéraire déjà paru, par lequel le réécrivain dompte les conséquences d'un événement au lieu de les subir. On verra que le braconnage ramuzien relève du paradoxe, car il consiste à exploiter au mieux certaines propriétés de l'écrit littéraire pour mieux en déjouer d'autres.

\section{Réécrire pour rafraîchir}

Ramuz s'attarde particulièrement sur son travail de réécriture dans les «Choses écrites pendant la guerre. 1939-194127», un journal rédigé en vue de la publication de ses CEuvres complètes chez Mermod et donc précisément au moment où il est pris dans une relecture massive et systématique de son œuvre. Le terme de «correction» y réfère au processus de «relecture crayon en main » à quelque stade de la genèse que ce soit. Il peut s'agir de la relecture d'un brouillon, d'un dactylogramme ou d'un jeu d'épreuves, comme d'une édition à rééditer. L'écrivain ne fait pas de distinction terminologique entre le travail de reprise effectué sur manuscrit pour préparer une rédaction ultérieure et la relecture de textes publiés destinés à préparer une édition prochaine. Il les envisage pourtant différemment. De son point de vue aussi, la relecture comporte certains risques. D'abord ces «révisions » absorbent le temps et l'énergie qui pourraient être consacrés au travail de «vision». Plus gravement, elles menacent la cohérence du texte, bâtie sur une intention de départ (à laquelle Ramuz réfère en tant qu'unité de ton) :

On voit qu'on ne change rien à un texte, même dans l'infime détail, sans que de proche en proche tout l'ensemble demande à être changé et qu'y ayant introduit autre chose, tout l'ensemble demande à être autre chose : le paragraphe décidant de la page, la page du chapitre, le chapitre du livre, la grammaire redressée influant sur la syntaxe, la syntaxe remise en place, à son tour, sur la composition, la composition sur l'idée même du livre $[\ldots]^{28}$.
Pour Ramuz, il y a peu de justification à ce geste épuisant, potentiellement dangereux, «nécessaire pourtant» :

Corrections commencées; le plus ingrat et déprimant travail; conscience de gâter souvent la phrase vivante - nécessaire pourtant 29 .

À quoi bon alors? Si le généticien s'engage à lutter contre le mirage téléologique qui lui ferait envisager chaque version comme meilleure que la précédente, il est clair que, du point de vue de l'écrivain, la réécriture ne se justifie pas sans l'espoir d'une amélioration ${ }^{30}$. Ramuz, qui use volontiers de métaphores vitalistes pour caractériser son travail (ainsi dans l'exergue celle, antithétique, des «cendres »), appelle «fraîcheur» la qualité qui motive la reprise d'un texte. Mais une telle propriété, celle d'un fruit dont la consommation n'attend pas, qui rapidement se périme, relance indéfiniment le geste de réécriture :

Peut-être qu'il y avait un peu de fraîcheur dans telle page écrite par vous [l'auteur lui-même], il y a bien longtemps : or on ne peut «perfectionner» la fraîcheur qu'en y ajoutant de la fraîcheur ${ }^{31}$.

La «fraîcheur» dont parle Ramuz évoque la dyade fraîcheur/ stéréotype de Roland Barthes, qui considère la première comme un antidote à la fatigue représentée par le second ${ }^{32}$. Barthes assigne au stéréotype (qui avant d'être une forme de discours fût une forme utilisée par les imprimeurs) une valeur doublement péjorative : il marque la reconduction d'une parole autre là où l'écrivain doit faire acte d'individualité, l'usure là où prime l'originalité. Le texte imprimé,

27. C. F. Ramuz, Journal 3, op. cit., p. 309-366.

28. C. F. Ramuz, Journal 3, op. cit., p. 331, entrée de novembre 1939.

29. C. F. Ramuz, Journal 2, op. cit., p. 93, entrée du 24 avril 1908 (au sujet de la mise au point de la première édition de Jean-Luc Persécuté).

30. On n'envisagera pas ce perfectionnement comme un résultat, mais comme une intention qui précède le travail de réécriture ; il est un vouloirmieux-dire qui se situe dans la tête de l'auteur, et non dans le texte (S. Pétillon, «Intentionnalité et révision à l'épreuve du manuscrit d'écrivain. Genèse de Génitrix de Mauriac», Langages, $\mathrm{n}^{\circ}$ 164, «La révision de texte : méthodes, outils et processus », 2006, p. 26-42).

31. C. F. Ramuz, Journal 3, op. cit., p. 330, entrée de novembre 1939. 32. R. Barthes, Roland Barthes par Roland Barthes, Euvres complètes, t. IV, Livres, textes, entretiens 1972-1976, éd. E. Marty, Paris, Éditions du Seuil, 2002 [1975], p. 666-667. 
fabriqué en série, souffre des mêmes défauts et appartiendrait pour Ramuz à la catégorie des produits langagiers usés. Le retravailler permet à l'écrivain de restaurer la singularité et l'origine d'une parole que son mode de circulation a désancré et, partant, de mettre le produit en relation avec l'acte qui l'a naguère réalisé, de rétablir l'actualité d'une énonciation qui se périme aussitôt dite. L'imaginaire langagier ramuzien d'un écrit caduc à revivifier stimule la lecture crayon en main, qui matérialise la réappropriation et la réactualisation du message par son énonciateur. Les motifs linguistiques ou poétiques de la réécriture sont secondaires relativement à cet imaginaire qui impose la réécriture par principe et qui surdétermine la représentation que l'écrivain se fait des constituants du procès énonciatif nécessairement impliqués par la réécriture : la situation, l'énonciateur, le temps et l'énonciataire.

\section{L'expérience de la réécriture comme ré-énonciation}

Lorsqu'il réécrit, Ramuz est cet écrivain répondant activement à l'auto-injonction de son propre texte que décrit Cavaillès :

L'écrivain peut-il ignorer devant son texte cette injonction enfouie dans l'écriture ? L'écrivain peut-il être simple lecteur de son œuvre? Lorsque l'écrivain relit son texte (même publié), sa lecture est pétrie de la nécessité de le transformer, d'actualiser ce hiéroglyphe gravé par un autre moi ; la genèse se poursuit bien dans l'œuvre («constante genèse» selon Blanchot) $[\ldots]^{33}$.

On peut caractériser le réécrivain comme un scripteur/ lecteur particulier, qui, dans la situation spécifique d'une re-publication, déjoue les conditions de la communication littéraire en régime de littérature-texte pour réactiver le lien auteur-scripteur. Ramuz témoigne du brouillage, pour lui, de ces instances dissociées par l'analyse autant que par le geste éditorial :

Le grand malheur, voyez-vous, pour un auteur, est qu'il soit un homme public; qu'il le devienne nécessairement, dès qu'il se mêle de «publier». Il a beau faire tout ce qu'il peut pour départager sa personne en deux moitiés dont l'une est l'homme, qu'il se réserve, l'autre l'auteur qu'il abandonne aux éléments et aux événements conjugués; la cloison qu'il voudrait étanche ne l'est guère. Publier suppose public : l'étymologie le veut ainsi et est dans son droit. L'écrivain excite l'opinion à se prononcer à son endroit (et l'écrivain est une espèce de neutre); mais l'homme est là quand même à une des extrémités du phénomène (si je puis dire) et n'arrive jamais à s'en séparer complètement ${ }^{34}$.

Le lien de production qui lie le scripteur (que Ramuz appelle «l'homme») à son texte, et qui pour lui persiste, a néanmoins été rompu par la publication. Le réactiver nécessite la réappropriation d'un objet que son statut d'œuvre littéraire a sédimenté aux yeux du public, dans sa forme et surtout dans son âge. De fait, lors d'une réécriture, le signal graphique est modifié après avoir reposé durant un temps plus ou moins long, de quelques semaines à plusieurs décennies. Le document a duré, mais le «je» et le contexte historique ne sont plus les mêmes; un écart s'est creusé qu'il s'agit pour Ramuz de négocier. L' «Avertissement» d'Aline justifie la réédition du roman par la permanence du caractère de son auteur, dans un geste destiné à lui permettre d'incarner, à ses yeux et à ceux des autres (l'éditeur, le public), malgré la menace d'anachronisme que le temps passé fait peser sur l'entreprise, l'actuel sujet du texte 35 :

J'étais un tout petit garçon quand je l'ai écrite [1'histoire d'Aline]; elle est pleine d'ingénuité. Mais peut-être bien reste-t-on un petit garçon toute sa vie; je suis assez de cet avis; et peut-être bien, d'autre part, avez-vous jugé que l'ingénuité n'est pas nécessairement un défaut 36 .

On envisagera de ce fait le temps comme une des contraintes majeures pesant sur la représentation que se fait Ramuz de

33. N. Cavaillès, «Génétique et dialogisme», Genesis, n 30, «Théorie : état des lieux », 2010, p. 193.

34. C. F. Ramuz, «Seconde lettre», Essais 2, op. cit., [1929], p. 180.

35. Résolution impossible si on en croit les propos de Roger Chartier en introduction du livre intitulé La Main de l'auteur et l'esprit de l'imprimeur, dans lequel il reprend des textes déjà parus séparément, qu'il présente comme une réécriture actualisante tout en admettant l'échec d'un tel projet : «Pour un auteur, même historien, se relire est toujours une épreuve. Les essais ici rassemblés ont été revus soigneusement [...]. Écrits aujourd'hui, ces textes seraient sans doute différents [...] » (R. Chartier, La Main de l'auteur et l'esprit de l'imprimeur, Paris, Gallimard, 2015, p. 19).

36. C.F. Ramuz, «Avertissement», op. cit. 
son geste 37 . La lecture/réécriture à visée ré-énonciative nécessite de composer avec la perception d'une épaisseur temporelle qu'il lui faut tenter de résoudre. Lorsqu'il se relit, Ramuz traduit d'une certaine manière l'espace en temps, l'écrit en écriture ; il envisage, sous les informations purement spatiales du produit, les paramètres temporels de sa production. La consistance temporelle ainsi mise à jour menace de faire obstacle à la réappropriation du texte. De fait, en dépit de la diversité des objets sur lesquels s'exerce la réécriture, on constate pour les romans Grasset une concordance entre les niveaux modifiés et l'âge des textes. Le volume des modifications et leur nature mettent en évidence que pour l'écrivain vaudois la durée de l'intervalle entre le temps de l'écriture et celui de la lecture/réécriture contribue à figer le texte. Les romans des débuts - Aline (1905), Jean-Luc Persécuté (1908) et Aimé Pache, peintre vaudois (1911) - font pour Grasset l'objet de modifications relativement peu nombreuses, très circonscrites et d'ordre stylistique. Le scénario éditorial évoqué plus haut amène cependant parfois Ramuz à reprendre pour Grasset un roman fraîchement paru chez Mermod : on observe alors le même genre de modifications stylistiques que dans les romans plus anciens, mais on en constate également d'autres, qui touchent à la composition du texte 38 . En plus d'un texte très remanié, la version Grasset de Farinet ou la fausse monnaie comporte par exemple une inversion des trois premiers chapitres de la version Mermod. On observe des révisions similaires dans la version Grasset d'Adam et Ève : l'organisation des chapitres finaux est changée et l'intrigue se dénoue différemment que dans l'édition originale. Les possibles multiples parmi lesquels le scripteur a effectué ses choix sont encore présents à sa mémoire : les déplacements effectués lors de la réécriture de Farinet ou la fausse monnaie prolongent par exemple une caractéristique de la préparation du roman, rédigé par fragments reliés ultérieurement ${ }^{39}$. La proximité chronologique du temps de l'écriture du livre et de celui de sa réécriture réduit le texte publié au statut d'excroissance accidentelle et provisoire d'une activité de préparation que le scripteur se représente toujours en cours : les réécritures de Farinet et la fausse monnaie et d'Adam et Ève ne sont pas effectuées à partir des éditions originales des deux romans mais des exemplaires des dactylogrammes qui les ont préparées (voir fig. 2 et 3). Aux yeux de Ramuz, ce que la publication ne fait pas, le temps par contre finit par s'en charger : il transforme ces écrits en «cendre froide» dont seuls quelques tisons donnent à l'écrivain matière à réécriture.

\section{Les motifs de l'amélioration : faire mieux «sentir»}

Contrairement à ce qu'a pu penser la critique, la représentation que se fait l'écrivain de son nouveau public ne l'emporte pas sur le critère qui semble déterminer, à ses yeux, la possibilité de se réapproprier une énonciation : l'âge du texte. L'ordre génétique des différentes versions (d'une édition de travail à celle qu'elle a préparée) met en évidence que le lieu de publication n'est pas décisif : lorsque Ramuz prépare ses Euvres complètes (1940-1941), qui sont destinées à un public suisse, il travaille à partir du texte Grasset lorsque l'édition originale est ancienne (voir fig. 4). La succession des versions permet également d'observer que, quand l'édition Grasset suit l'édition originale de quelques mois (quand autrement dit la différence d'âge est peu pertinente), c'est systématiquement l'édition originale que choisit Ramuz, à nouveau indépendamment de son lieu de parution (voir fig. 5). On observe également cette manière de faire en amont, au moment de la préparation des textes pour Grasset : l'écrivain revient à l'édition originale lorsqu'elle est récente, au mépris, lorsqu'elle existe, d'une version intermédiaire publiée en France ${ }^{40}$. Aux yeux de

37. C. Leblay, «Introduction», dans C. Leblay et G. Caporossi (dir.), Écriture et enregistrement, Louvain-la-Neuve, Académia Bruylant, 2014, p. 11.

38. Le Garçon savoyard, dont l'édition chez Grasset suit de peu celle de Mermod et présente des modifications peu nombreuses, est un cas particulier.

39. Pour les genèses de Farinet ou la fausse monnaie, voir V. Nicollier, «"Il n'y a pas de beauté, là où elle ne se communique pas, là où elle n'est pas mise en circulation" », dans C. F. Ramuz, Romans 8, Euvres complètes, t. XXVI, Genève, Slatkine, 2013, p. 383-408. Concernant celle d'Adam et Ève, voir D. Maggetti, «Fatalité de la malédiction», dans C.F. Ramuz, Romans 9, Euvres complètes, t. XXVII, Genève, Slatkine, 2013, p. 3-31.

40. C'est le cas de Si le soleil ne revenait pas : pour préparer l'édition Grasset de 1939, Ramuz préfère l'édition originale de 1937 à celle des Nouvelles littéraires de 1938. De même, l'édition Grasset d'Adam et Ève est travaillée à partir du dactylogramme qui prépare l'édition originale de 1932 en dépit de la version $N R F$ qui paraît la même année quelques mois plus tard. 


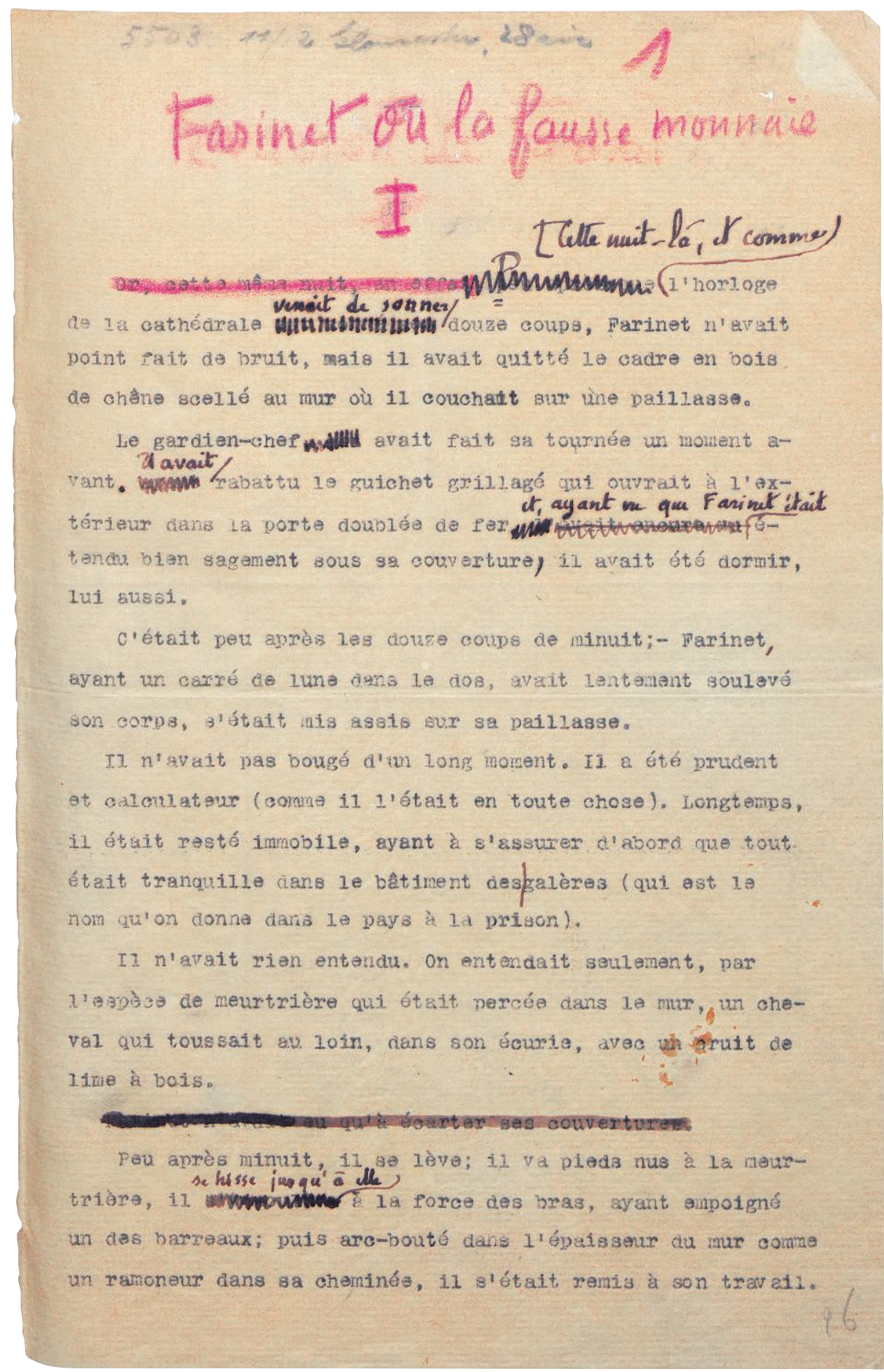

Fig. 2 : Feuillet du dactylogramme de Farinet ou la fausse monnaie établi pour préparer l'édition originale du roman (Mermod, 1932) et utilisé pour travailler sa réédition chez Grasset (1932). Le nouveau manuscrit définitif que Ramuz envoie à l'éditeur parisien ( 225 feuillets recto reliés) se compose de pages du dactylogramme abondamment réécrites, déplacées et de nombreux feuillets manuscrits. Il s'ouvre sur une des spectaculaires inversions de chapitres qui font la particularité de la version Grasset du roman : le récit débute désormais sur ce qui constituait, dans l'édition originale établie d'après le dactylogramme, le début du chapitre II (Fonds privé) 


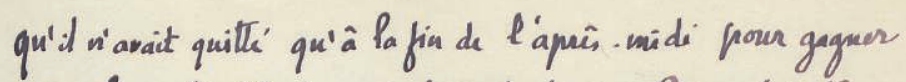
unfin ln punts d'herle quis descundent ven Borng-Saint.

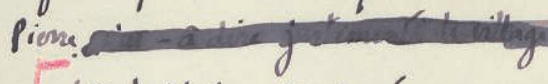

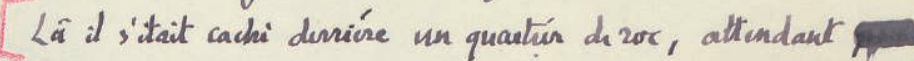

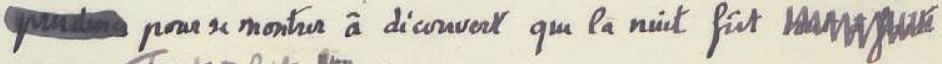
orn Fout a foit lin venue.

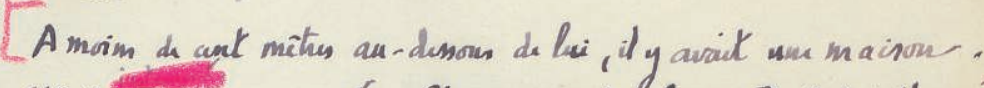

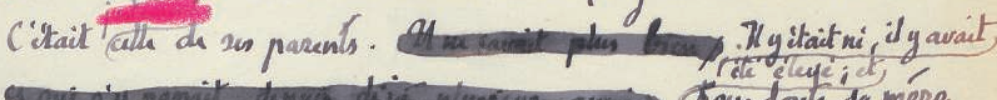

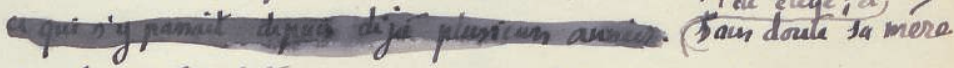

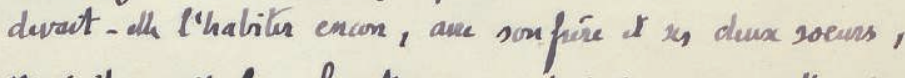
mais il y avait hien longtemps qu' il dait saus nouralles d'cuxx. Con il itait parti pous. Mieges, pren apres la mot dis son pere qu'on avaik tiouvi un jon la téte pacance aupied d'une paroi

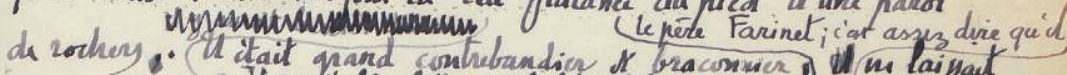

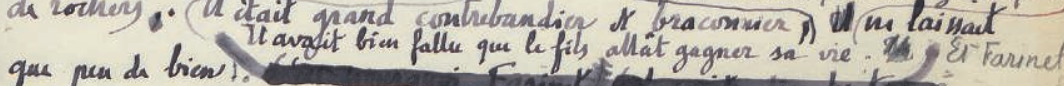

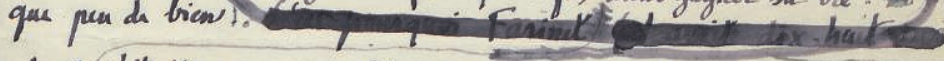

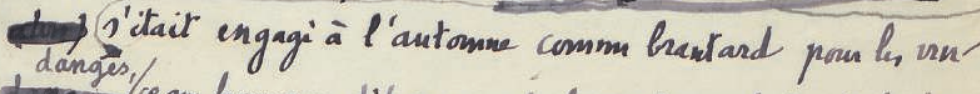

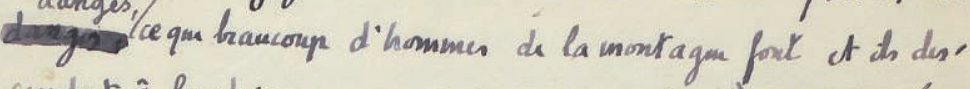

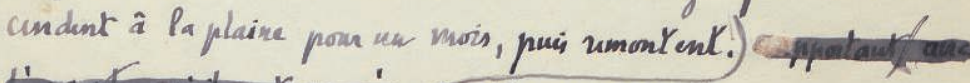
tiangent quith oxt gages (Er Farinet itait dnandu ans cusc,

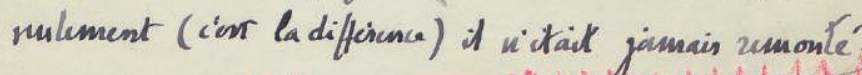
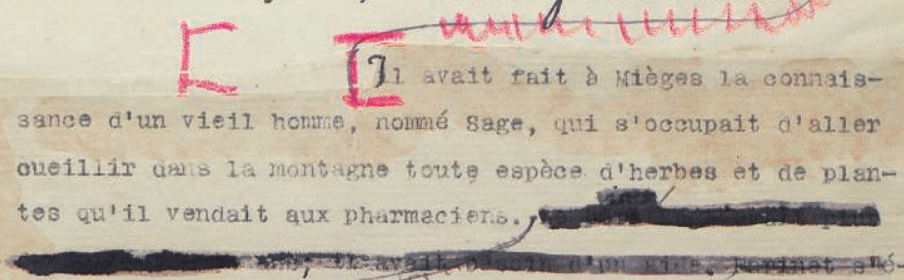

Fig. 3 : De nombreux collages témoignent du travail de mise en page auquel se livre Ramuz pour assembler, dans un manuscrit définitif qu'il destine à Grasset, les feuillets manuscrits aux feuillets dactylographiés. Le feuillet 14 (chap. II) comporte ainsi, à la suite d'une partie manuscrite, un passage découpé dans le feuillet 20 de l'exemplaire du dactylogramme dont se sert l'écrivain (chap. III) (Fonds privé) 


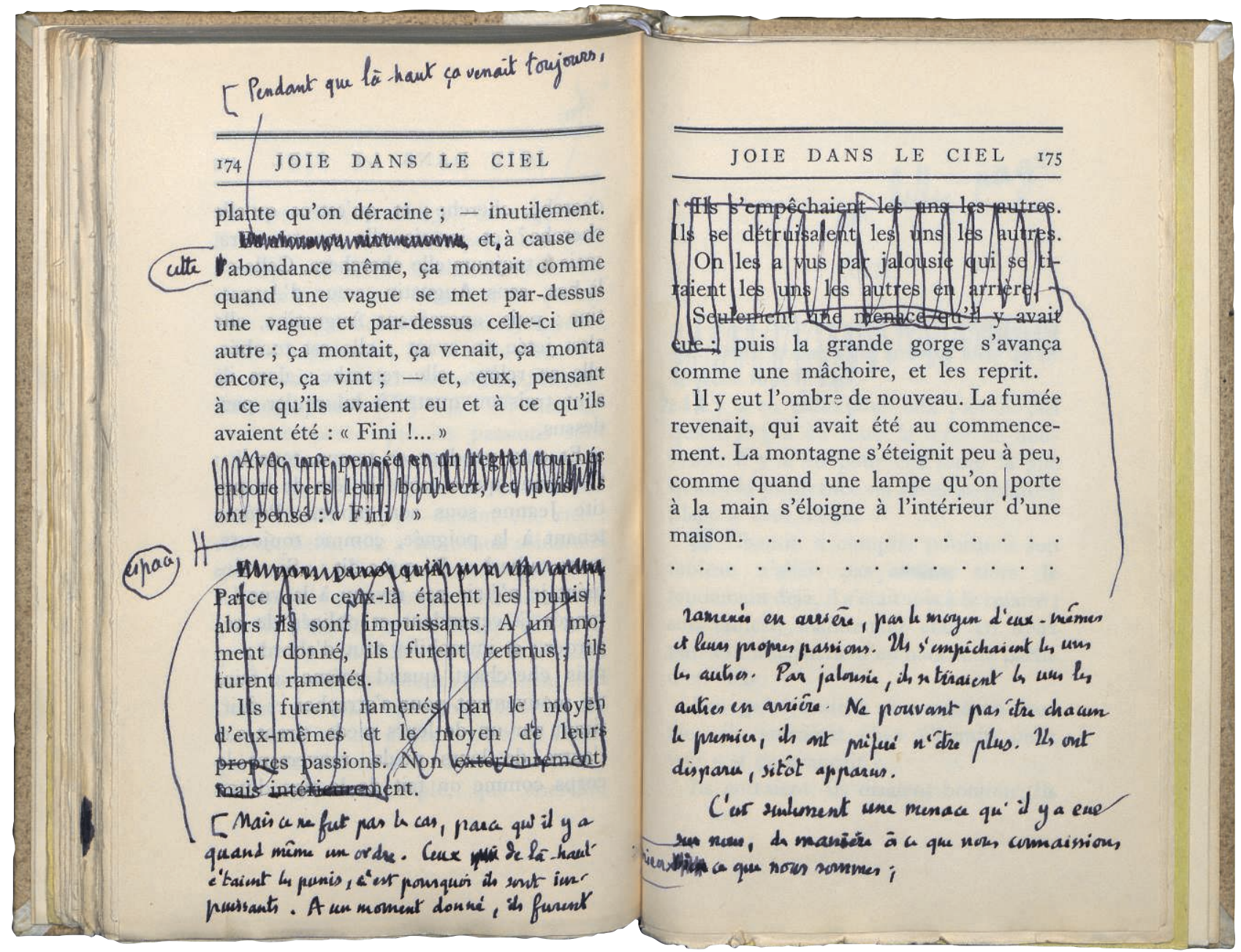

Fig. 4 : Pages d'un exemplaire de l'édition Grasset de Joie dans le ciel (1925) corrigé de la main de Ramuz en vue de la réédition du roman dans les Euvres complètes de Mermod (Terre du ciel, 1942) (Bibliothèque cantonale et universitaire, Lausanne)

Ramuz, le pays de publication n'est pas un critère déterminant à l'heure de choisir la version qu'il va réécrire : son choix se porte d'abord sur une version récente, puis, lorsqu'elle fait défaut, sur l'édition originale.

Envisager le destinataire effectif du livre ne suffit donc pas pour comprendre ce qui se joue pour Ramuz lors de réécritures dont on a vu qu'elles étaient destinées avant tout à la réappropriation d'un propos déjà-énoncé. Dans le cas de la production écrite qui nous occupe, le «destinataire» du texte recouvre également une autre entité : le scripteur lui-même, qui durant tout le processus de réécriture est son seul lecteur, cette «double locution» inhérente au travail de l'écriture dont Grésillon et Lebrave observent les traces sur les manuscrits 41 . Suivant ce point de vue, il est plus productif d'envisager la réécriture comme un lieu de tension entre une énonciation pour soi et une énonciation pour autrui. Plus qu'aux attentes qu'il prête à son lectorat, le réécrivain est confronté à la non-coïncidence entre le sens produit par son texte sur le lecteur qu'il est aujourd'hui avec l'intention du scripteur qu'il était hier, ou le souvenir de cette intention :

Farinet doit être complètement récrit : vous [Poulaille] devinez bien ce que le livre a d'intime : il faudrait seulement que le lecteur le sentît mieux.

41. A. Grésillon et J.-L. Lebrave, «Linguistique et génétique des textes : un décalogue», Le Français moderne, «Tendances actuelles de la linguistique française », Paris, CILF, 2008, p. 37-49. 


\begin{tabular}{|c|c|c|c|c|c|}
\hline Aline & EO 1905 & $\longrightarrow$ & G 1927 & $\longrightarrow$ & $\begin{array}{l}\text { M } 1940 \\
=\text { Kundig } 1934\end{array}$ \\
\hline Jean-Luc persécuté & EO 1908 & $\longrightarrow$ & \multicolumn{2}{|c|}{ Georg $1920 \rightarrow$} & G $1930 \longrightarrow$ M 1940 \\
\hline Aimé Pache, peintre vaudois & EO 1911 & $\longrightarrow$ & \multicolumn{2}{|l|}{ M 1940} & $=$ La Guilde du livre 1945 \\
\hline La Guérison des maladies & EO 1917 & $\longrightarrow$ & G 1924 & $\longrightarrow$ & M 1941 \\
\hline Les Signes parmi nous & EO 1919 & $\longrightarrow$ & G 1931 & $\longrightarrow$ & M 1941 \\
\hline Terre du ciel & EO 1921 & $\longrightarrow$ & \multicolumn{2}{|c|}{$\longleftrightarrow$} & $\begin{array}{l}\text { M } 1941 \\
\text { Gonin } 1941\end{array}$ \\
\hline La Beauté sur la terre & EO 1927 & $\longrightarrow$ & $\begin{array}{l}\text { G } 1928 \\
\text { M } 1941\end{array}$ & $\longrightarrow$ & Les Feuillets bleus 1935 \\
\hline Farinet ou la fausse monnaie & D 1932 & $\longrightarrow$ & $\begin{array}{l}\text { EO } 1932 \\
\text { G } 1932\end{array}$ & $\longrightarrow$ & $\begin{array}{l}\text { M } 1941 \\
=\text { Edition des Bibliophiles franco-suisses } 1938\end{array}$ \\
\hline Adam et Ève & D 1932 & $\longrightarrow$ & $\begin{array}{l}\text { EO } 1932 \\
\text { G } 1933\end{array}$ & $\longrightarrow$ & M 1941 \\
\hline Derborence & EO 1934 & $\longrightarrow$ & $\begin{array}{l}\text { G } 1936 \\
\text { M } 1941\end{array}$ & & \\
\hline Si le soleil ne revenait pas & EO 1937 & $\longrightarrow$ & $\begin{array}{l}\text { G } 1939 \\
\text { M } 1941\end{array}$ & $\longrightarrow$ & La Guilde du livre 1940 \\
\hline Le Garçon savoyard & EO 1936 & $\begin{array}{l}\longrightarrow \\
\longrightarrow\end{array}$ & $\begin{array}{l}\text { G } 1937 \\
\text { M } 1941\end{array}$ & & \\
\hline La Guerre aux papiers & EO 1942 & $\longrightarrow$ & G 1945 & & \\
\hline
\end{tabular}

Fig. 5 : Le tableau ci-dessus met en évidence la place occupée par les textes Grasset (G) dans les réécritures ramuziennes; il indique d'une part les versions utilisées pour préparer la publication des romans chez l'éditeur parisien, d'autre part la place des versions Grasset dans les réécritures ultérieures. Lorsque l'édition originale (EO), au moment de la réécriture, date de quatre ans ou plus, on observe que c'est la version Grasset qui sert à préparer l'édition ultérieure, principalement celle des Euvres complètes chez Mermod (M). (On notera que l'édition Grasset d'Aimé Pache, peintre vaudois fait à double titre figure d'exception, puisqu'elle est postérieure à celle de Mermod et que pour la préparer, Ramuz revient à l'édition originale malgré son «ancienneté».) Par contre, lorsque Ramuz retravaille un roman dont l'édition originale est plus récente, comme c'est le cas des volumes publiés par Mermod à partir de 1926, il préfère repartir de l'édition originale ou d'un exemplaire du dactylogramme qui l'a préparé (D).

[J]e suis un petit peu embarrassé, parce qu'elle [la fin de La Beauté sur la terre] doit l'être [désordonnée]. L'affaire serait donc de le faire bien sentir au lecteur, et c'est à quoi je vais tâcher de m'employer ${ }^{42}$.

Dans ces deux extraits de lettres adressées aux gens de Grasset, la première à Poulaille et la seconde à Tisné, Ramuz affirme effectuer des ajustements pour son lecteur, mais sans doute faut-il considérer que l'image qu'il se fait de son lecteur a les traits familiers de sa propre réception. Cette dernière, suivant son parti pris anti-intellectualiste, est guidée par une esthétique du senti et de l'émotion plutôt que du compris et de l'explication. Faire sentir au mieux, c'est se poser la question des effets produits par son texte lors de la relecture. Lorsqu'il se relit crayon en main, Ramuz ne cherche pas à corriger des fautes, mais il s'assigne, comme lecteur, la tâche de tester ces effets, avant d'ajuster, comme scripteur, ce qui doit l'être.

42. Lettre à Poulaille du 15 mai 1932 (C. F. Ramuz, Lettres 1919-1947, Étoy, Les Chantres, p. 257); lettre à Tisné du 23 février 1928 (Fonds privé); dans les deux cas, c'est moi qui souligne. 
Plus le texte est ancien, plus sa lecture revêt les traits d'une réception «ordinaire», délestée du souvenir de sa genèse 43 .

La réécriture ramuzienne est ainsi mue par deux moteurs, dont les mécanismes concourent à la même action mais peuvent se contrarier : désir de se réapproprier l'expérience de la parole dont l'écrit n'est qu'une trace et volonté constante d'ajuster un ressenti de lecteur actuel avec une intention parfois très ancienne. D'un côté, la tentation de réaliser au mieux une intention originaire, d'un autre, la pulsion d'être à nouveau le sujet du texte. Ces deux mobiles peuvent conduire à des changements en cascade qui menacent le même sur le fond duquel la réécriture peut s'exercer :
Fini la dernière correction de «Posés...». Est-ce que ce sera d'ailleurs la dernière? Tout dépend d'un effort de volonté. Il faut qu'on se dise : C'est fini, je n'y touche plus ; - sans quoi on voit que de correction en correction on referait le livre entier ${ }^{44}$.

43. Localement et sur des points précis, Ramuz tient évidemment compte des remarques de la critique. Lorsque il réécrit Adam et Ève pour Grasset, il modifie copieusement un chapitre vivement critiqué par des lecteurs prestigieux de la $N R F$ (par exemple J. Paulhan), ce qui donne l'impression qu'il y souscrit. Cependant, une lecture attentive de ces modifications révèle que la différence n'est pas dans le sens général du passage qui était incriminé, mais dans le renforcement de certains éléments mal compris (V. Nicollier, «Une énonciation sous influence...», art. cité, p. 5-22). 44. C. F. Ramuz, Journal 3, op. cit., p. 328, entrée du 16 février 1943.

VAlentine NiCOLlier SARAILlon est chargée de cours à l'Université de Lausanne. Elle a participé à l'édition critique et génétique des Euvres complètes de C.F. Ramuz, où elle a entre autres édité des volumes de Nouvelles et morceaux et le roman Farinet ou la fausse monnaie. Ses recherches en génétique portent en particulier sur les rapports entre genres et brouillons : «La forme et l'accident. Le genre comme élément variant du processus de production» (Recto verso, $\mathrm{n}^{\circ} 4,2008$ ) et, en collaboration avec R. Mahrer, «Les brouillons font-ils texte?» (Faire texte. Frontière textuelles et opérations de textualisation, Presses universitaires de FrancheComté, 2015). Doctorante associée au séminaire «Manuscrit - Linguistique - Cognition» de l'ITEM (CNRS-ENS) dirigé par R. Mahrer et B. Vauthier, elle prépare une thèse sur la notion de «ton» dans les poétiques de R. Pinget et de C.F. Ramuz.

valentine.nicollier@unil.ch 


\section{Réécrire pour énoncer à nouveau : l'exemple de Ramuz chez Grasset}

C.F. Ramuz (1878-1947) est un réécrivain systématique. Cet article propose de mettre en perspective les réécritures qu'il effectue dès 1924 pour son éditeur parisien Grasset en envisageant d'une part les conditions de ce geste de réécriture en contexte d'énonciation éditoriale, d'autre part l'effet, sur la pratique de la réécriture, de la représentation que s'en fait le réécrivain. Ce double axe de réflexion permet d'observer que la finalité que Ramuz assigne à la réécriture n'est pas de corriger des fautes ni d'adapter sa langue à un nouveau lectorat, mais de réactiver le lien de production qui unit le scripteur à son texte, un lien dont la publication a entériné la rupture. L'hypothèse d'un imaginaire de la réécriture comme une re-énonciation met en évidence les principaux enjeux que revêt ce geste aux yeux de l'écrivain. Ces enjeux, la problématique du temps de l'écriture et de l'âge de l'écrit ou celle d'une forme particulière de double locution qui confère au scripteur la fonction de premier lecteur de son livre, déterminent ainsi la finalité et le type de réécritures effectuées par l'écrivain.

C.F. Ramuz is a systematic rewriter. This article will put into perspective the rewritings he carried out as early as 1924 for his Parisian publisher Grasset. We will consider on the one hand the conditions of this rewriting gesture, in the context of an editorial enunciation, and on the other, the effect on the writing practice and on its representation for the writer. This double axis of reflection helps to observe that the finality that Ramuz assigns to rewriting is not to correct mistakes nor to adapt his language to a new readership, but to reactivate the production link that unites the scriptor and his text, a connection broken by the publication. The hypothesis of a rewriting imaginary, as a re-enunciation, highlights the issues mainly at stake for the writer in this gesture. These issues, the problematic of writing time and the age of writing or that of a particular form of double locution conferring to the scriptor the function of first reader of his own book, thus determine the finality and the type of rewritings carried out by the writer.

C.F. Ramuz (1878-1947) ist ein systematischer Überarbeiter seiner Texte. Der vorliegende Artikel widmet sich den seit 1924 für seinen Pariser Verleger Grasset vorgenommenen Überarbeitungen, indem einerseits die Bedingungen der Überarbeitung im Kontext der Verlegerarbeit und andererseits die Wirkung des Umschreibens auf die Vorstellungswelt des Autors erwogen werden. Dank dieser doppelten Perspektive lässt sich beobachten, dass Ramuz mit seiner Überarbeitung nicht den Zweck verfolgt, Fehler zu korrigieren oder seine Sprache einem neuen Lektorat anzugleichen, sondern den produktiven Nexus wiederherzustellen, der den Schreibenden mit seinem Text verbindet, einen Nexus, der durch die Publikation unterbrochen wurde. Die Hypothese der Neu-Schreibung als Neu-Artikulation zeigt die grundlegenden Herausforderungen, die dieser Gestus für den Schriftsteller mit sich bringt. Diese Herausforderungen, die Problematik der Zeit des Schreibens und des Alters des Geschriebenen oder die spezielle Form der doppelten Rede verleihen dem Autor die Funktion des ersten Lesers seines Buches, und sie bestimmen so Zweck und Typus der vom Schriftsteller vorgenommenen Überarbeitungen.
C.F. Ramuz (1878-1947) es un reescritor sistemático. Este artículo se propone poner en perspectiva las reescrituras que efectúa a partir de 1924 para su editor parisiense Grasset, considerando, por una parte, las condiciones de este gesto de reescritura en un contexto de enunciación editorial y, por la otra, el efecto que tiene en la práctica de la reescritura, la manera en que el reescritor se la representa. Este doble eje de reflexión permite observar que la finalidad que Ramuz le asigna a la reescritura no es la de corregir los errores ni de adaptar su lengua a un nuevo público lector, sino la de reactivar la relación de producción que vincula al scriptor con su texto, cuya ruptura ha sido refrendada por la publicación. La hipótesis de un imaginario de la reescritura como una re-enunciación pone en evidencia las diversas significaciones que el escritor le atribuye. Estas significaciones, la problemática del tiempo de la escritura y de la edad de lo escrito o la de una forma particular de doble locución que confiere al scriptor la función de primer lector de su libro, determinan de este modo la finalidad y el tipo de reescrituras efectuadas por el escritor.

C. F. Ramuz (1878-1947) é por sistema um re-escritor. Este artigo examina as revisões que ele executa a partir de 1924 para o seu editor parisiense Grasset, considerando por um lado as condições desse gesto em termos de enunciação editorial e por outro o efeito que isso tem sobre a representação que de si faz o re-escritor. Este duplo eixo de reflexão permite observar que Ramuz não atribui à reescrita a finalidade de corrigir erros nem de adaptar a sua linguagem a um novo público, mas sim de reativar o elo produtivo que une o escritor ao seu texto, elo que a publicação tinha rompido. A hipótese de um imaginário da reescrita como uma reenunciação destaca questões essenciais deste gesto aos olhos do escritor. O momento da escrita, a idade da obra escrita, essa espécie de dupla locução que confere ao escritor a função de primeiro leitor do seu livro, são questões problematizantes que interferem na finalidade e no tipo de intervenções efectuadas pelo autor.

C.F. Ramuz (1878-1947) è un riscrittore sistematico. L'articolo si propone di mettere in prospettiva le riscritture effettuate dal 1924 per il suo editore parigino Grasset considerando, da un lato, le condizioni di questo gesto di riscrittura in un contesto di enunciazione editoriale, dall'altro, l'effetto, sulla pratica della riscrittura, della rappresentazione che se ne fa il riscrittore. Questo doppio asse di riflessione permette di osservare che la finalità assegnata da Ramuz alla riscrittura non è la correzione di errori né l'adattamento della sua lingua a nuovi lettori, bensì la riattivazione del legame di produzione che unisce lo scrittore al suo testo, legame la cui rottura è ratificata dalla pubblicazione. L'ipotesi di un immaginario della riscrittura come ri-enunciazione mette in evidenza le principali implicazioni che questo gesto riveste agli occhi dello scrittore. Queste implicazioni - la problematica del tempo della scrittura e dell'età dello scritto o quella di una forma particolare di doppia locuzione che conferisce allo scrittore la funzione di primo lettore del suo libro - determinano così la finalità e il tipo delle riscritture effettuate dallo scrittore. 